\title{
超支化聚酯对聚乙二醇非等温结晶行为的影响
}

\author{
刘晶如“徐 洁董鸿超俞 强 \\ (常州大学材料科学与工程学院, 江苏常州 213164)
}

\begin{abstract}
摘要: 利用差示扫描量热法(DSC)、偏光显微镜(POM)以及傅里叶变换红外 $(F T I R)$ 光谱研究了一种超支化聚 酯(HBP)对聚乙二醇(PEG)非等温结晶行为的影响, 用 Ozawa 法、Jeziorny 法和莫志深法对非等温结晶动力学 进行了分析. 结果表明: PEG 和 HBP/PEG 的非等温结晶过程可用 Ozawa 和莫志深动力学方程描述, 与 Jeziorny动力学方程不符; 超支化聚酯的加入改变了 PEG 的结晶成核和生长机理, 对 PEG 的结晶有一定的延 缓作用. 超支化聚酯中的羰基与 PEG 的端羟基形成的氢键以及超支化聚酯自身较大的分子体积和高度支化的 结构所导致的位阻效应是超支化聚酯延缓PEG 结晶的主要原因.
\end{abstract}

关键词: 聚乙二醇; 非等温结晶; 超支化聚酯; 差示扫描量热法

中图分类号: 0643

\section{Effect of Hyperbranched Polyester on the Non-Isothermal Crystallization Behavior of Polyethylene Glycol}

\author{
LIU Jing-Ru* XU Jie DONG Hong-Chao YU Qiang \\ (School of Materials Science and Engineering, Changzhou University, Changzhou 213164, Jiangsu Province, P. R. China)
}

\begin{abstract}
The effect of hyperbranched polyester (HBP) on the non-isothermal crystallization behavior of polyethylene glycol (PEG) was studied by differential scanning calorimetry (DSC), polarizing microscopy (POM), and Fourier transform infrared (FTIR) spectroscopy. The non-isothermal crystallization data for PEG and HBP/PEG were analyzed by the Ozawa, Jeziorny, and Mo Zhi-Shen methods. The results indicate that the non-isothermal crystallization processes of PEG and HBP/PEG may be described using the kinetic equations of Ozawa and Mo, but do not agree with the Jeziorny equation. The addition of HBP affected the crystallization process and crystal form of pure PEG, and slowed the rate of crystallization. The hydrogen bond formed between the carbonyl group of HBP and the terminated-hydroxyl group of PEG, and the larger size and highly branched structure of HBP are the main reasons for the slower crystallization.
\end{abstract}

Key Words: Polyethylene glycol; Non-isothermal crystallization; Hyperbranched polyester; Differential scanning calorimetry

\section{1 引言}

聚乙二醇(PEG)是一种结构规整且柔性良好的 端羟基聚醚, 有多种优良特性而被广泛应用. ${ }^{2}$ PEG 作为硝酸酯增塑聚醚(NEPE)推进剂的常用粘合剂, 与硝酸酯的相容性好, 与硝化甘油的最大混溶比可 达 4 以上, 有利于推进剂能量的提高. ${ }^{2-4}$ 以 PEG 为粘
合剂的 NEPE推进剂目前遇到的最大问题是力学性 能较差, 这主要是由于 PEG 极易结晶, 而结晶是相 分离的驱动力, PEG 的结晶会降低所制备的推进剂 的应变能力和改变其与含能增塑剂的互溶能力, 从 而影响推进剂的力学性能. 5,6

以 PEG 为粘合剂相的网络结构(即 PEG 基聚氨

Received: October 20, 2011; Revised: December 12, 2011; Published on Web: December 20, 2011.

"Corresponding author. Email: ruruliu1028@163.com; Tel: +86-13813669351.

The project was supported by the National Natural Science Foundation of China (20674032).

国家自然科学基金(20674032)资助项目

(C) Editorial office of Acta Physico-Chimica Sinica 
酯弹性体)是 NEPE 推进剂的基础, 其力学性能的好 坏直接决定了推进剂力学性能的优劣. ? 超支化聚合 物具有独特的支化分子结构, 分子之间无缠结, 并 且含有大量的端基, 因而表现出高溶解度、低粘度、 高的化学反应活性等许多线形聚合物所不具备的 优异性能. ${ }^{8-17}$ 前期我们将少量超支化聚酯(HBP)引 入到 PEG 基聚氨酯弹性体中, 发现弹性体的拉伸强 度和断裂伸长率均提高了 2 倍以上, 取得了较好的 增强增韧效果..$^{18}$ 本文进一步采用差示扫描量热法 (DSC) 研究 PEG 和 $\mathrm{HBP} / \mathrm{PEG}$ 的非等温结晶过程, 用 三种动力学方法(Ozawa 法、Jeziorny 法、莫志深法) 分析其非等温结晶动力学, 探讨 HBP 对 PEG 结晶行 为的影响.

\section{2 实验部分}

\section{1 实验原料}

PEG, 纯度 $98 \%$, 数均分子质量 6000 , 熔点为 $57^{\circ} \mathrm{C}$, 分析纯, 国药集团化学试剂有限公司, $100{ }^{\circ} \mathrm{C}$ 下减压脱水至无气泡后使用; HBP, 自制. PEG 和 HBP 的质量比为 100:6, HBP 为直接加入, PEG 和 $\mathrm{HBP}$ 熔融后调匀.

超支化聚酯的合成: 以三羟甲基丙烷(TMP)为 核, 2,2-二羟甲基丙酸(bis-MPA)为支化单体, 按 1:3 的摩尔比加入三口烧瓶中, 再加入占原料总质量比 为 $0.3 \%$ 的催化剂对甲苯磺酸, 在氮气保护下, $120^{\circ} \mathrm{C}$ 熔融搅拌 $40 \mathrm{~min}$, 升温至 $140{ }^{\circ} \mathrm{C}$, 抽真空, 继续反应 $4 \mathrm{~h}$, 得到无色块状固体, 为第一代的超支化聚酯. 然 后继续加入 2 倍于初始量的二羟甲基丙酸和对应量 的对甲苯磺酸, 重复前面的过程即可制得第二代的
超支化聚酯. 依次类推, 重复前面的过程就可以得 到第三代超支化聚酯(HBP-OH).

超支化聚酯的改性及提纯: 在装有回流冷凝 管、搅拌器、分水器、温度计并通氮气保护的三口烧 瓶中加入一定量的 HBP-OH、过量的十八酸以及质 量分数为 $0.3 \%$ 的对甲苯磺酸, 加热至回流, 反应 3 $\mathrm{h}$, 抽真空至体系无水泡冒出, 得到的棕色蜡状固体 (HBP).

在 $500 \mathrm{~mL}$ 烧杯加入 $250 \mathrm{~mL}$ 冰醋酸和 $50 \mathrm{~g}$ 改性 后的 $\mathrm{HBP}$, 然后加热至 $60{ }^{\circ} \mathrm{C}$ 左右, 使其溶解, 用玻 璃棒充分搅拌后, 得到混浊体系, 在室温下冷却 5-6 h, HBP 凝固在冰醋酸上层, 将其取出后捣碎, 用大量去离子水冲洗, 吹干后将其加热熔融, 趁热 加入足量的去离子水使醋酸尽量去除, 将产物捣碎 后放入三口烧瓶中, 加热熔融后摚拌, 分别用水洜 和油泵抽真空至体系无气泡生成. 在 $100{ }^{\circ} \mathrm{C}$ 下, 将 最后所得产物置于真空干燥箱中 $24 \mathrm{~h}$, 取出冷却至 室温, 得到提纯的 HBP, 为白色蜡状固体.

反应式和产物结构如图 1 所示.

\section{2 实验仪器及测试条件}

非等温结晶动力学测试在美国 Perkin Elmer 公 司生产的 DSC-7 型差示扫描量热仪上进行. 样品质 量约为 $10 \mathrm{mg}$, 氮气气氛, 氮气流量为 $40 \mathrm{~mL} \cdot \mathrm{min}^{-1}$. 首先快速升温至 $75^{\circ} \mathrm{C}$ 使样品熔融, 恒温 $10 \mathrm{~min}$ 以 消除热历史, 然后分别以 $1 、 2 、 3 、 4 、 5^{\circ} \mathrm{C} \cdot \mathrm{min}^{-1}$ 的降 温速率降至室温, 得到一系列不同降温速率下的非 等温 DSC 结晶曲线.

HBP、PEG 及 HBP/PEG 置于研针中与溴化钾研 磨均匀后用压片机压片, 在美国尼高力公司生产的
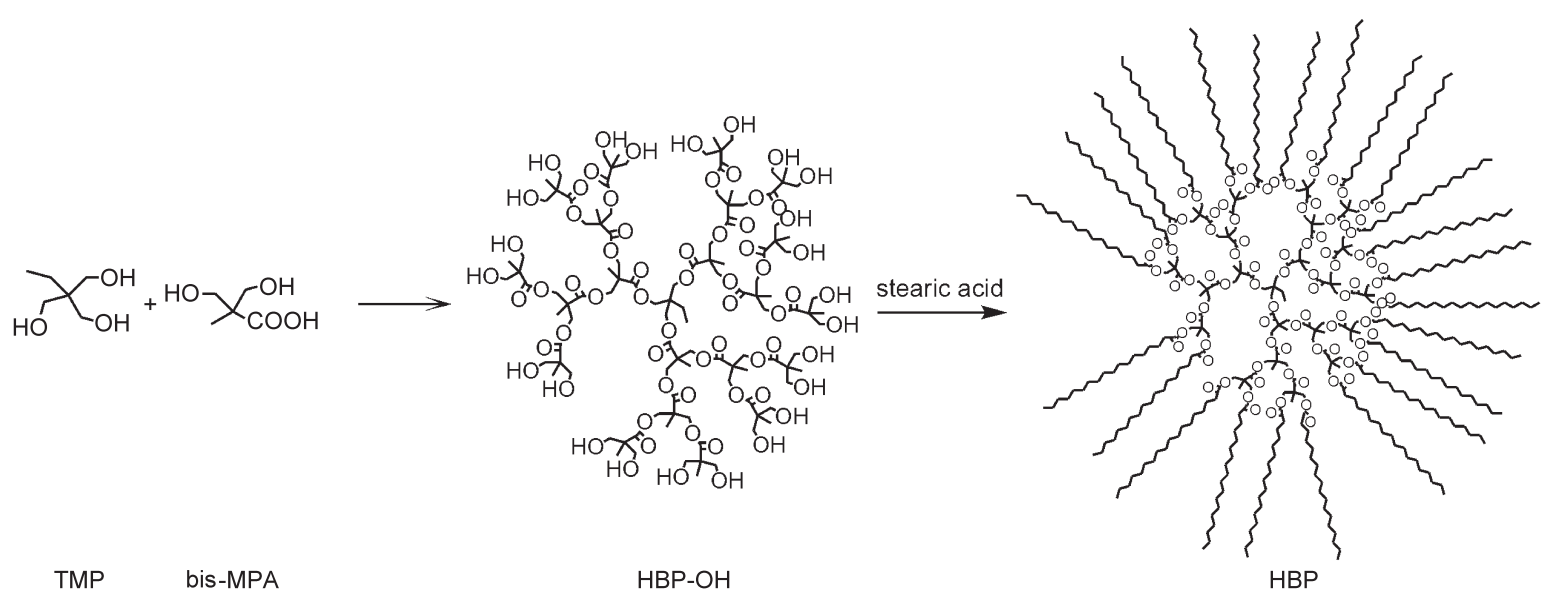

图 1 反应式及产物结构

Fig.1 Reaction formula and chemical structures of products

TMP: tri(hydroxymethyl) propane; bis-MPA: 2,2-dimethylol propionic acid; HBP: hyperbranched polyester 
Nicolet 370 型傅里叶变换红外光谱仪上进行红外测 试, 扫描次数 64 次, 分辨率 $0.4 \mathrm{~cm}^{-1}$, 扫描范围 $500-4000 \mathrm{~cm}^{-1}$.

端羟基超支化聚酯的溶剂为氝代二甲基亚砜, HBP 的溶剂为気代氯仿, 在瑞士 Bruker 公司生产的 AVANCE III $500 \mathrm{MHz}$ 核磁仪上进行 ${ }^{1} \mathrm{H}$ 核磁共振 (NMR)测试.

采用日本岛津公司生产的 DSC-60 差示扫描量 热仪来表征超支化聚酯的熔点, 样品质量约为 $5 \mathrm{mg}$, 氮气气氛, 氮气流量为 $20 \mathrm{~mL} \cdot \mathrm{min}^{-1}$. 为消除热历史, 采用二次升温的办法, 一次升温速率 $10^{\circ} \mathrm{C} \cdot \mathrm{min}^{-1}$, 从室温到 $100^{\circ} \mathrm{C}$, 恒温 $5 \mathrm{~min}$ 以消除热历史, 然后以 $10^{\circ} \mathrm{C} \cdot \mathrm{min}^{-1}$ 的降温速率降至 $0{ }^{\circ} \mathrm{C}$, 再以 $10^{\circ} \mathrm{C} \cdot \mathrm{min}^{-1}$ 的升温速率升温至 $100^{\circ} \mathrm{C}$.

将 PEG 及 $\mathrm{HBP} / \mathrm{PEG}$ 于 $70^{\circ} \mathrm{C}$ 熔融后, 迅速降温 至 $25^{\circ} \mathrm{C}$ 结晶制片. 在江南光学公司生产的 XPT-7 型 偏光显微镜上观察样品的结晶形态.

\section{3 结果与讨论}

\subsection{HBP 的结构表征}

HBP-OH 和 HBP 的红外光谱如图 2 所示. 可以 看出, HBP-OH 在 $3400 \mathrm{~cm}^{-1}$ 附近的峰为醇羟基的特 征吸收峰, 2980 和 $2880 \mathrm{~cm}^{-1}$ 附近的峰为亚甲基的不 对称和对称伸缩振动的特征吸收峰, $1738 \mathrm{~cm}^{-1}$ 处为 酯基的特征吸收峰, 说明 HBP-OH 是端羟基超支化 聚酯; 用十八酸改性后, HBP 在 $3400 \mathrm{~cm}^{-1}$ 处的羟基 峰消失, 2918 和 $2848 \mathrm{~cm}^{-1}$ 附近亚甲基吸收峰的强度 明显增强, 且在 $721 \mathrm{~cm}^{-1}$ 附近出现了 4 个以上亚甲 基相连 $\left(-\left(\mathrm{CH}_{2}\right)_{n}-, n \geq 4\right)$ 时的亚甲基平面摇摆振动 的特征吸收峰, 说明改性后超支化聚酯的亚甲基数 目明显增加, 超支化聚酯端羟基已被长链烷基取代.

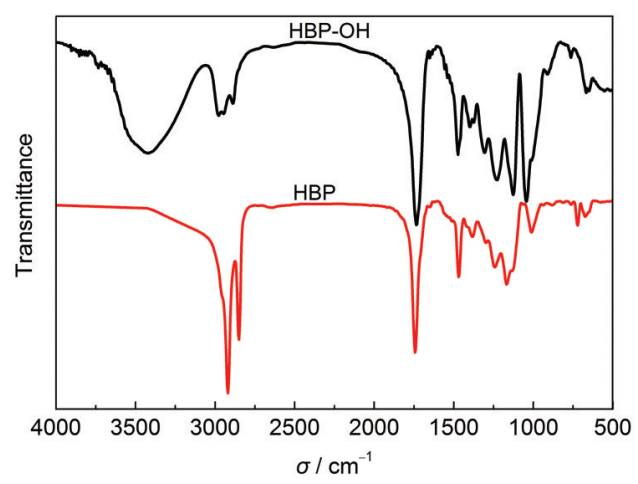

图 $2 \mathrm{HBP}-\mathrm{OH}$ 和 HBP 的红外(IR)光谱图 Fig.2 Infrared (IR) spectra of HBP-OH and HBP

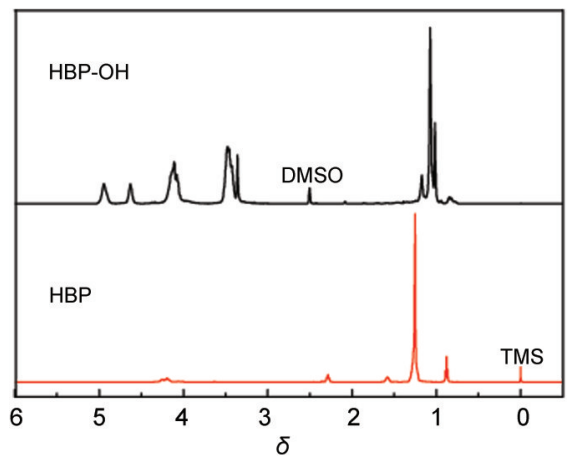

图 $3 \mathrm{HBP}-\mathrm{OH}$ 和 $\mathrm{HBP}$ 的 ${ }^{1} \mathrm{H}$ NMR 谱

Fig.3 ${ }^{1} \mathrm{H}$ nuclear magnetic resonance (NMR) spectra of HBP-OH and HBP

DMSO: dimethyl sulfoxide; TMS: trimethyl silane

图 3 为 $\mathrm{HBP}-\mathrm{OH}$ 和 $\mathrm{HBP}$ 的 ${ }^{1} \mathrm{H}$ NMR 谱, 可以看 出, HBP-OH 的端羟基质子峰出现在 4.96 和 4.64 处, 在 4.0-4.2 处为与酯基相连的亚甲基质子峰, 3.3-3.6 为与端羟基相连的亚甲基质子峰, 0.9-1.2 为甲基的 质子峰; 用十八酸改性后, 羟基在 4.96 和 4.64 处的 质子峰消失, 4.2 处为与酯基相连的亚甲基质子峰, 与端羟基相连的亚甲基在 3.3-3.6 处的质子峰消失, 用十八酸酯化后与酯基相连的第一个亚甲基的质 子峰出现在 2.3 处, 酯化后与酯基相连的第二个亚 甲基质子峰出现在 1.6 处, 脂肪族链的亚甲基质子 峰在 1.2-1.4 处, 甲基质子峰在 0.9 处. 这说明十八酸 已通过酯化反应成功接到超支化聚酯的端基上.

已有实验结果 ${ }^{16}$ 表明, 聚合物的末端结构对聚 合物的结晶性能有很大影响. 图 4 为 HBP 的 DSC 升 温曲线, 可以看出 $\mathrm{HBP}$ 在 $35^{\circ} \mathrm{C}$ 左右存在一个明显 的吸热峰, 归属于脂肪族长链端基结晶的熔融, 即 所合成的超支化聚酯是结晶聚合物.

\subsection{PEG 及 HBP/PEG 的非等温结晶过程}

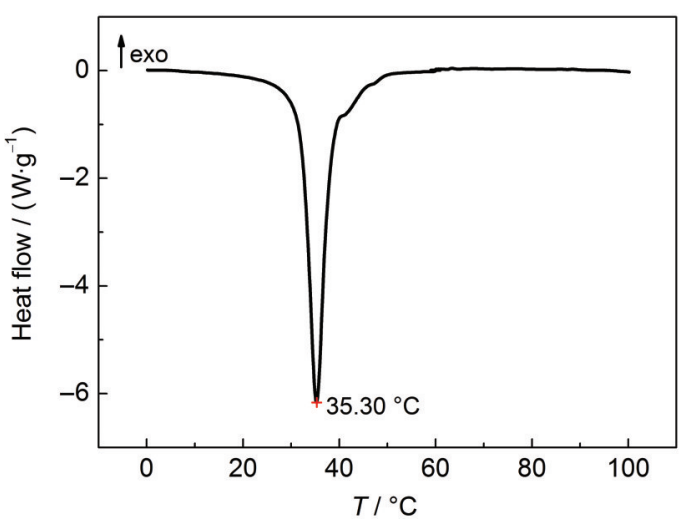

图 4 HBP 的 DSC 曲线

Fig.4 DSC curve of HBP 


\subsection{1降温速率对结晶温度的影响}

图 5 为不同降温速率下 $\mathrm{PEG}$ 和 $\mathrm{HBP} / \mathrm{PEG}$ 的 $\mathrm{DSC}$ 曲线. 可以看出, 结晶温度都随着降温速率增 大而降低. 这是因为降温速率增大时, 结晶温度下 降很快, 高分子链在高温下来不及作规则排列, 结 晶热效应在较低温度下显现, 结晶平衡需要在较宽 温度范围才能达到.

\subsection{2 降温速率对结晶时间的影响}

图 6 是相对结晶度 $\alpha(t)$ 与结晶时间 $t$ 的关系, 相 对结晶度 $\alpha(t)$ 可由公式(1)计算而得:

$$
\alpha(t)=\frac{X_{\mathrm{c}}(T)}{X_{\mathrm{c}}\left(T_{\infty}\right)}=\frac{\int_{T_{0}}^{T} \frac{\mathrm{d} H_{\mathrm{c}}(T)}{\mathrm{d} T} \mathrm{~d} T}{\int_{T_{0}}^{T_{\infty}} \frac{\mathrm{d} H_{\mathrm{c}}(T)}{\mathrm{d} T} \mathrm{~d} T}
$$

式中, $T_{0}$ 和 $T_{\infty}$ 分别代表初始结晶的温度及完全结晶 时的温度, $X_{\mathrm{c}}(T)$ 和 $X_{\mathrm{c}}\left(T_{\infty}\right)$ 分别代表温度 $T$ 时及完全结 晶时的结晶度, $H_{\mathrm{c}}(T)$ 代表温度 $T$ 时的结晶焓. 利用 温度 - 时间转换公式 $t=\left(T_{0}-T\right) / \beta$ 可以计算出达到某 一相对结晶度时所需的时间 ( $\beta$ 为降温速率). 由图 6

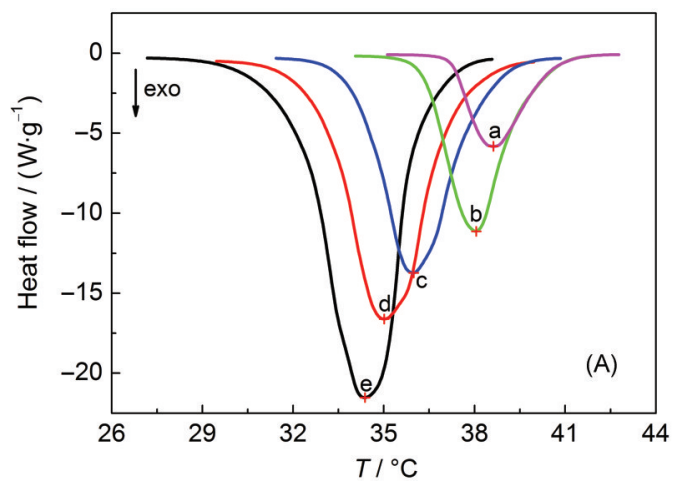

表 1 PEG 和 HBP/PEG 的半结晶时间 $\left(t_{1 / 2}\right)$ Table 1 Half-crystallization time $\left(t_{1 / 2}\right)$ of PEG and HBP/PEG

\begin{tabular}{ccc}
\hline \multirow{2}{*}{$\beta /\left({ }^{\circ} \mathrm{C} \cdot \mathrm{min}^{-1}\right)$} & \multicolumn{2}{c}{$t_{1 / 2} / \mathrm{min}$} \\
\cline { 2 - 3 } & PEG & HBP/PEG \\
\hline 1 & 3.972 & 4.405 \\
2 & 1.834 & 2.358 \\
3 & 1.562 & 1.596 \\
4 & 1.215 & 1.054 \\
5 & 0.866 & 0.867 \\
\hline
\end{tabular}

可以看出, 开始很短一段时间内相对结晶度增长缓 慢, 之后随温度和时间增加而呈线性急剧增长, 最 后增长速度逐渐变缓. 通过图 6 可以确定出用于表 征结晶速率的半结晶时间 $t_{1 / 2}$, 结果列于表 1 . 从表 1 可以看出, 降温速率小于 $3{ }^{\circ} \mathrm{C} \cdot \mathrm{min}^{-1}$ 时, $\mathrm{HBP} / \mathrm{PEG}$ 的 $t_{1 / 2}$ 值比 PEG 的大; 当降温速率大于 $3^{\circ} \mathrm{C} \cdot \mathrm{min}^{-1}$ 时, $\mathrm{HBP} / \mathrm{PEG}$ 的半结晶时间与 $\mathrm{PEG}$ 的半结晶时间差不 多, 甚至小于 PEG 的半结晶时间.

\section{3 非等温结晶动力学}

非等温结晶动力学过程较为复杂, 目前的理论 分析和数据处理方法都不能准确地对这一过程进

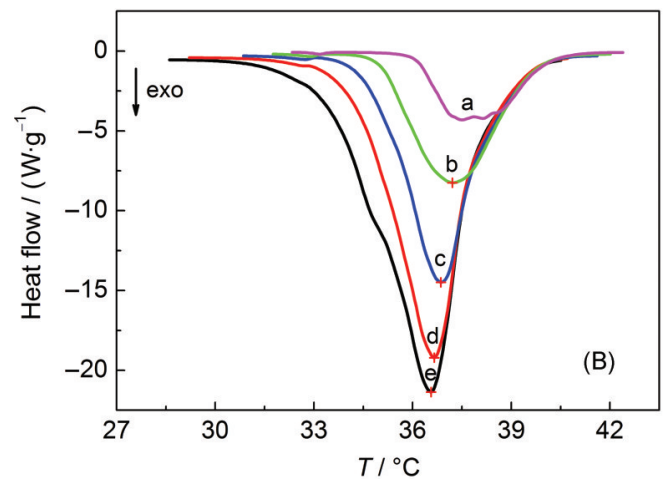

图 5 不同降温速率 $(\beta)$ 下 PEG (A)及 HBP/PEG (B) 结晶过程的 DSC 曲线

Fig.5 DSC curves of the crystallization process of PEG (A) and HBP/PEG (B) at different cooling rates $(\beta)$ $\beta /\left({ }^{\circ} \mathrm{C} \cdot \min ^{-1}\right)$ : (a) 1 ; (b) 2 ; (c) 3 ; (d) 4 ; (e) 5
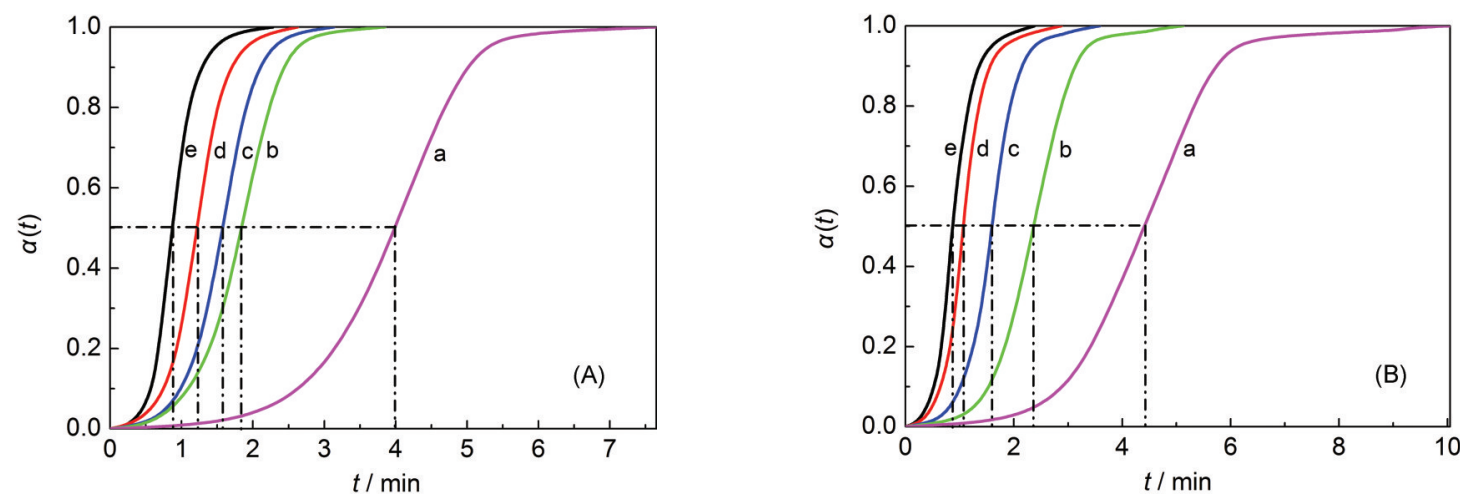

图 6 不同降温速率下 PEG (A)及 HBP/PEG (B)相对结晶度 $(\alpha(t))$ 随时间的变化

Fig.6 Plots of relative crystallinity $(\alpha(t))$ vs time of PEG (A) and HBP/PEG (B) at different cooling rates $\beta /\left({ }^{\circ} \mathrm{C} \cdot \min ^{-1}\right):$ (a) 1 ; (b) 2 ; (c) 3 ; (d) 4 ; (e) 5 
行描述, 较为常用的处理方法有 Ozawa法、Jeziorny 法以及莫志深法等.

\subsection{1 用 Ozawa方程处理的非等温结晶动力学}

Ozawa 方程 ${ }^{19}$ 是一种常用的解析聚合物非等温 结晶动力学参数的方法, 它从聚合物结晶的成核和 生长出发, 导出了等速升温或等速降温时的结晶动 力学方程:

$$
\lg [-\ln (1-\alpha(T))]=\lg k(T)-n \lg \beta
$$

公式(2)中, $\alpha(T)$ 为温度 $T$ 时的相对结晶度; $k(T)$ 为与 温度有关的冷却结晶函数; $n$ 为 Avrami 指数. 在给定 温度的条件下, 以 $\lg [-\ln (1-\alpha(T))]$ 对 $\lg \beta$ 作图, 得图 7 所示的斜率为 $-n$ 的直线.

由图 7 得不同温度时的 $n$ 值, 结果如表 2 所示. Avrami 指数 $n$ 反映了高聚物成核机理和生长方式的 情况, 整数 $n$ 等于成核过程的时间维数和晶体生长 的空间维数之和. 成核过程有均相成核和异相成核 两种. 均相成核有时间依赖性, 时间维数为 1 , 而异 相成核与时间无关, 其时间维数为 0 . 由表 2 数据可 见, $n$ 值为非整数, 且不同温度下的 $n$ 值和冷却结晶 函数 $\lg k(T)$ 值均相差较大, 说明 $\mathrm{PEG}$ 和 $\mathrm{HBP} / \mathrm{PEG}$ 的 结晶机理复杂, 不同温度下存在着不同的结晶机 制. 随着温度的升高, PEG 体系的 Avrami 指数 $n$ 逐 渐增大, 数值在 1-5 之间, 说明随着温度的升高, PEG 晶体生长方式由一维增长逐渐发展到三维球 晶生长; 而 $\mathrm{HBP} / \mathrm{PEG}$ 体系 Avrami 指数 $n$ 变化幅度不 大, 平均值为 0.979 , 说明其成核方式为异相成核, 生 长方式为针状晶体的一维生长, 这主要是由于 HBP 的端基为长链烷基, 存在结晶性, 结晶的端基可起 到异相成核剂的作用. 超支化聚酯的加入改变了 PEG 晶体的生长方式.

\subsection{2 用 Jeziorny方程处理的非等温结晶动力学}

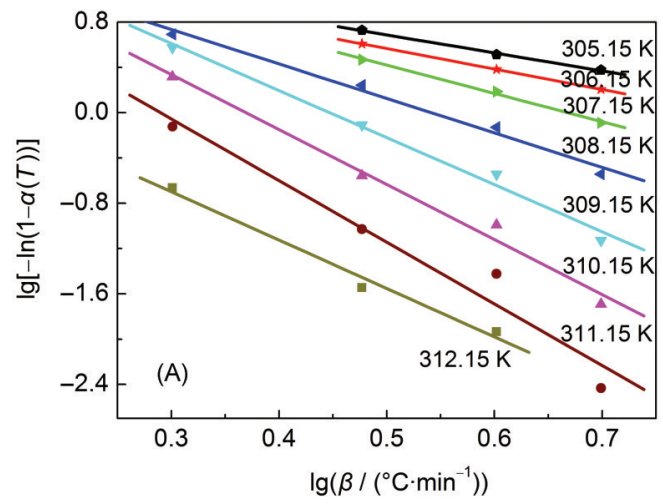

表 2 不同温度下 PEG 和 HBP/PEG 的 $n$ 和 $\lg k(T)$ 值 Table 2 Avrami exponent $n$ and $\lg k(T)$ of PEG and HBP/ PEG at different temperatures

\begin{tabular}{cccccc}
\hline \multirow{2}{*}{$T / \mathrm{K}$} & \multicolumn{2}{c}{ PEG } & & \multicolumn{2}{c}{ HBP/PEG } \\
\cline { 2 - 3 } \cline { 5 - 6 } & $n$ & $\lg k(T)$ & & $n$ & $\lg k(T)$ \\
\hline 305.15 & 1.592 & 1.484 & & 0.716 & 1.008 \\
306.15 & 1.810 & 1.472 & & 0.514 & 0.809 \\
307.15 & 2.500 & 1.670 & & 0.661 & 0.806 \\
308.15 & 3.045 & 1.649 & & 0.929 & 0.810 \\
309.15 & 4.154 & 1.857 & & 0.990 & 0.597 \\
310.15 & 4.857 & 1.794 & & 1.268 & 0.337 \\
311.15 & 5.444 & 1.578 & & 1.392 & -0.061 \\
312.15 & 4.271 & 0.583 & & 1.359 & -0.591 \\
\hline
\end{tabular}

$n$ : Avrami exponent; $k(T)$ : cooling function related to temperature

Jeziorny 法 $^{20}$ 是直接把 Avrami 方程推广应用于 解析等速变温 DSC 曲线的方法, 其实质是把结晶过 程先当作等温结晶过程来处理, 然后再对动力学参 数 $Z$ 进行修正.

Avrami 方程:

$$
(1-\alpha(T))=\exp (-Z t)
$$

可写成如下的线性形式:

$$
\lg [-\ln (1-\alpha(T))]=\lg Z+m \lg t
$$

公式(3)和(4)中, $Z$ 为复合结晶速率常数; $m$ 为 Ozawa 指数, 与成核机理和形成晶体的形态有关. 以 $\lg [-\ln (1-\alpha(T))]$ 对 $\lg t$ 作图, 从直线斜率得 $m$, 从截距 求得 $Z$. 由这种方法求出的 $Z$ 随 $\beta$ 而变化, 考虑到 $\beta$ 的 影响, 用公式(5)对其进行校正:

$$
\lg Z_{\mathrm{c}}=(\lg Z) / \beta
$$

Jeziorny 把 $Z_{\mathrm{c}}$ 作为表征非等温结晶动力学的参数.

图 8 为用 Jeziorny 方程处理的 PEG 和 HBP/PEG 结晶动力学关系曲线. 由图 8 可以看出, $\lg [-\ln (1-$ $\alpha(T)$ ]与 $\lg t$ 在低结晶度时线性关系较好, 而在高结晶 度时产生了线性偏离. 这是因为当结晶度达到一定

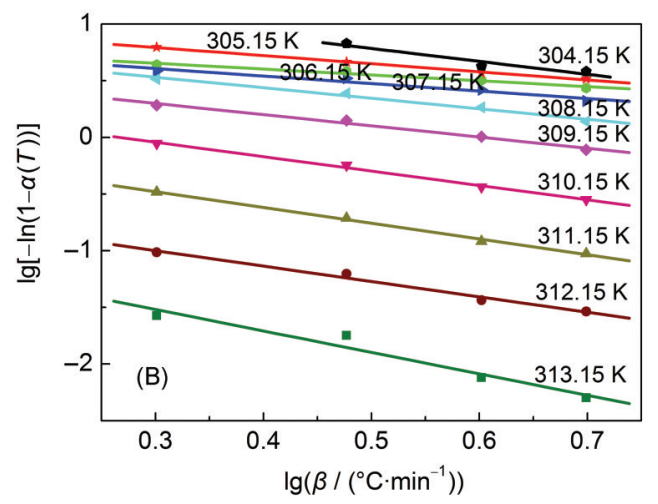

图 7 不同温度下 PEG (A)及 HBP/PEG (B) 结晶过程的 $\lg [-\ln (1-\alpha(T))]$ 与 $\lg \beta$ 的关系

Fig.7 $\lg [-\ln (1-\alpha(T))]$ vs $\lg \beta$ relationship for the crystallization process of PEG (A) and HBP/PEG (B) at different temperatures 

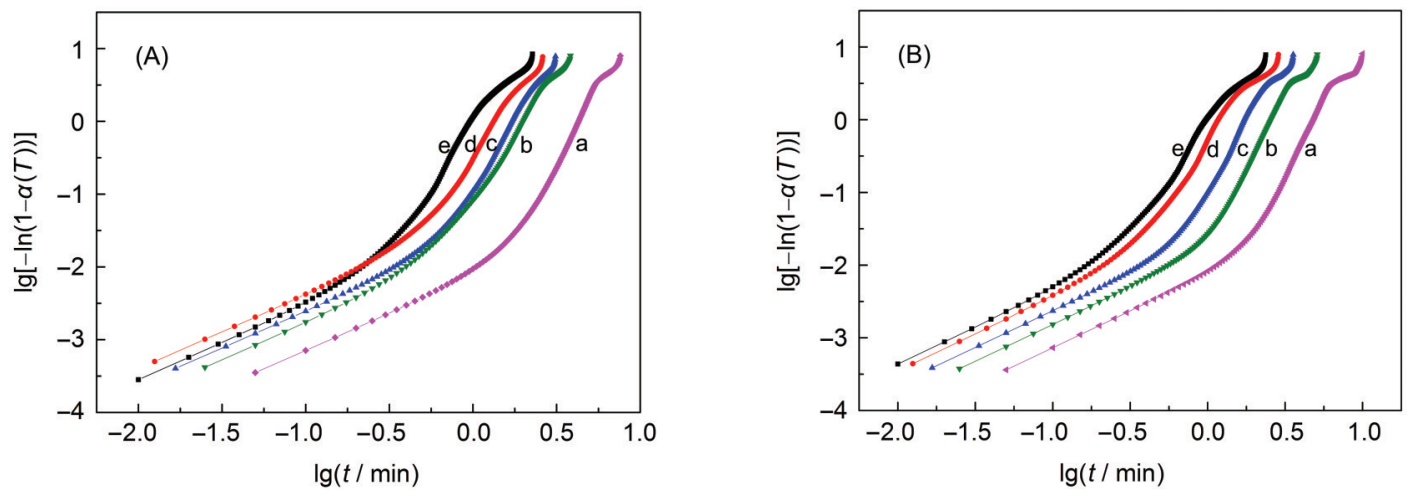

图 8 不同降温速率下 PEG (A)及 HBP/PEG (B) 结晶过程的 $\lg [-\ln (1-\alpha(t))]$ 与 $\lg t$ 的关系

Fig.8 $\lg [-\ln (1-\alpha(T))] v s \lg t$ relationship for the crystallization process of PEG (A) and HBP/PEG (B) at different cooling rates

$\beta /\left({ }^{\circ} \mathrm{C} \cdot \min ^{-1}\right)$ : (a) 1 ; (b) 2 ; (c) 3 ; (d) 4 ; (e) 5

程度时, 体系粘度增大, 链段运动困难, 结晶受扩散 控制, 二次结晶导致了这种线性偏离.

\subsection{3 用莫志深法处理的非等温结晶动力学}

莫志深等 ${ }^{21-23}$ 提出了一种解析结晶动力学参数 的新方法, 将 Avrami 方程和 Ozawa 方程结合, 得到 如下方程:

$$
\lg \beta=\lg F(T)-a \lg t
$$

公式(6)中, $F(T)=[K(T) / Z]^{1 / m}, a=n / m, n$ 和 $m$ 分别是 Avrami 指数和 Ozawa 指数, $F(T)$ 表示单位结晶时间 内体系要达到某一相对结晶度时所必须选取的降 温速率值, 即样品在一定结晶时间内达到某一结晶 度时的难易程度.

图 9 为在 4 个选定的相对结晶度下, $\lg \beta$ 与 $\lg t$ 的 关系曲线. 很明显, $\lg \beta$ 与 $\lg t$ 有较好的线性关系, 由 直线的斜率和截距求出的 $a$ 和 $F(T)$ 列于表 3 . 由表 3 可见, 相同试样、不同结晶度 $\alpha$ 下的 $a$ 变化不大, 随着 相对结晶度的提高, PEG 和 HBP/PEG 的 $F(T)$ 值均逐 渐增大, 说明其结晶速率随着相对结晶度的提高而

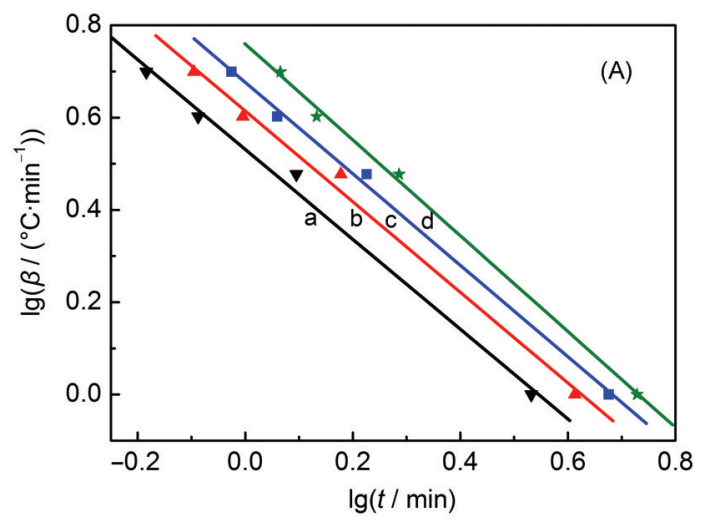

逐渐变慢; 比较表 3 的数据发现, 在相同的相对结晶 度下, 添加了超支化聚酯的 PEG 其 $F(T)$ 值均小于纯 PEG 的 $F(T)$ 值. $F(T)$ 值的减小说明 HBP/PEG 在单位 结晶时间内达到某一结晶度所必须选取的降温速 率比纯 PEG 的小, 需要给予足够的结晶时间才能使 其在单位结晶时间内达到某一结晶度, 表明超支化 聚酯的加入延缓了 PEG 的结晶. 聚合物的结晶过程 包括晶核生成和晶体生长两个步骤, 因此结晶速率 应该包括晶核生成速率、晶体生长速率以及由它们 共同决定的结晶总速率. 超支化聚酯在 PEG 中起到 异相成核剂的作用, 促进成核, 但是对 PEG 的结晶 总速率却起着延缓作用.

\subsection{HBP 对PEG 结晶过程影响的原因分析}

\subsubsection{HBP 自身的非等温结晶过程}

图 10 为不同降温速率下 $\mathrm{HBP}$ 的非等温结晶 DSC 曲线, 可以看出, 随着温度的降低, 在不同的降 温速率下均出现了一个较大的结晶放热峰, 对应于 长链端烷基有序排列而造成的结晶. 采用 Ozawa方

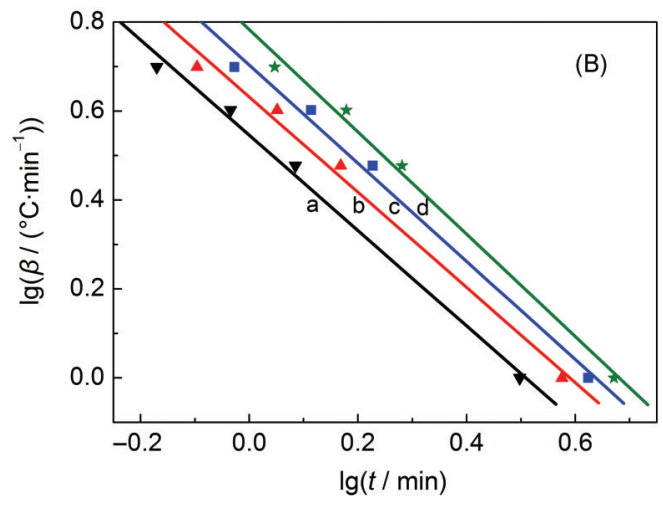

图 9 不同相对结晶度时 PEG (A)及 HBP/PEG (B)结晶过程的 $\lg \beta$ 与 $\lg t$ 的关系

Fig.9 $\lg \beta$ vs $\lg t$ relationship for the crystallization process of PEG (A) and HBP/PEG (B) at different relative crystallinity $\alpha$ : (a) 0.2 ; (b) 0.4 ; (c) 0.6 ; (d) 0.8 
表3 用莫志深法计算的 PEG 和 HBP/PEG 非等温结晶 动力学参数

Table 3 Non-isothermal crystallization parameters of PEG and HBP/PEG obtained by Mo Zhi-Shen method

\begin{tabular}{|c|c|c|c|c|}
\hline \multirow{2}{*}{$\alpha$} & \multicolumn{2}{|r|}{ PEG } & \multicolumn{2}{|c|}{ HBP/PEG } \\
\hline & $a$ & $F(T) /\left({ }^{\circ} \mathrm{C} \cdot \mathrm{min}^{-1}\right)$ & $a$ & $F(T) /\left({ }^{\circ} \mathrm{C} \cdot \min ^{-1}\right)$ \\
\hline 0.2 & 1.071 & 3.516 & 0.974 & 3.396 \\
\hline 0.4 & 1.070 & 4.285 & 0.981 & 4.111 \\
\hline 0.6 & 1.103 & 5.047 & 0.990 & 4.742 \\
\hline 0.8 & 1.149 & 6.067 & 1.038 & 5.741 \\
\hline
\end{tabular}

程处理其非等温结晶动力学, 在给定温度的条件 下, 以 $\lg [-\ln (1-\alpha(T))]$ 对 $\lg \beta$ 作图, 得到如图 11 所示 的斜率为 $-n$ 的直线. 由图 11 得不同温度时的 $n$ 值, 结果如表 4 所示. 封麟先等 ${ }^{24}$ 认为, $n$ 值的大小代表 了聚合物熔体结晶时结晶生长点的数目. HBP 在不 同温度下的 Avrami 指数 $n$ 均小于 0.5 , 说明其成核方 式为异相成核, 生长方式为针状晶体的一维生长, 且为受限结晶.

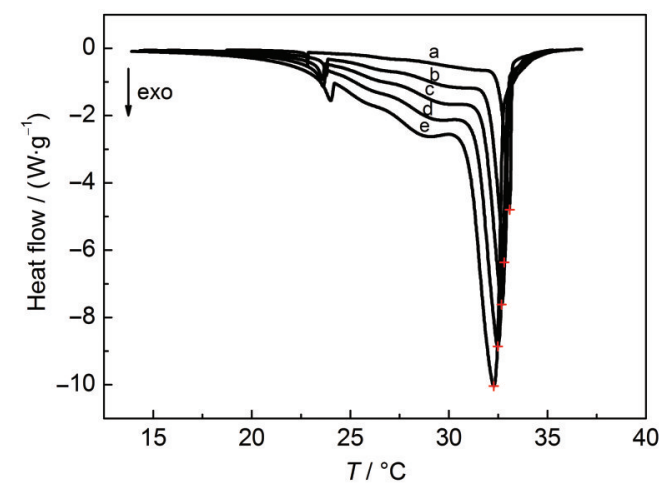

图 10 不同降温速率下 HBP 结晶过程的 DSC 曲线

Fig.10 DSC curves of the crystallization process of HBP at different cooling rates $\beta /\left({ }^{\circ} \mathrm{C} \cdot \mathrm{min}^{-1}\right):$ (a) 1 ; (b) 2 ; (c) 3 ; (d) 4 ; (e) 5

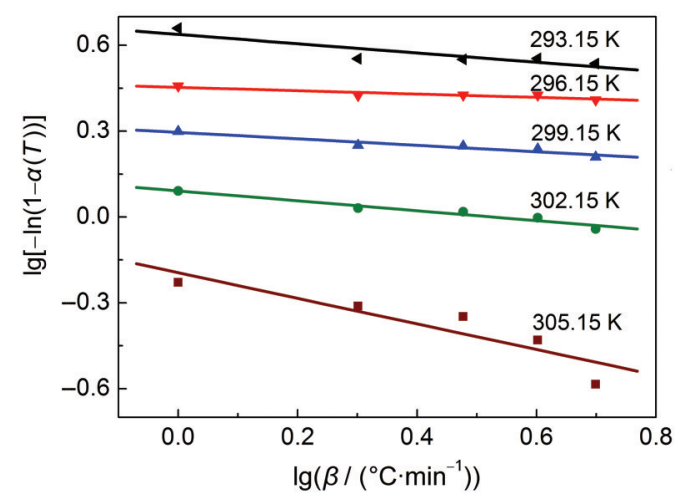

图 11 HBP 结晶过程的 $\lg [-\ln (1-\alpha(T))]$ 与 $\lg \beta$ 的关系

Fig.11 $\lg [-\ln (1-\alpha(T))] v s \lg \beta$ relationship for the crystallization process of HBP
表4 不同温度下 HBP 的 $n$ 和 $\lg k(T)$ 值

Table 4 Avrami exponent $n$ and $\lg k(T)$ of HBP at different temperatures

\begin{tabular}{ccc}
\hline$T / \mathrm{K}$ & $n$ & $\lg k(T)$ \\
\hline 293.15 & 0.161 & 0.638 \\
296.15 & 0.058 & 0.453 \\
299.15 & 0.113 & 0.296 \\
302.15 & 0.173 & 0.091 \\
305.15 & 0.447 & -0.195 \\
\hline
\end{tabular}

\subsubsection{PEG 及 HBP/PEG 的结晶形态}

图 12 为在相同放大倍数的偏光显微镜下拍得 的 PEG 和 $\mathrm{HBP} / \mathrm{PEG}$ 的结晶形态, 可以看出, PEG 晶 体呈现出球晶所特有的黑十字消光图像, 同时成核 并以相同速率生长的晶界面是一个平面; $\mathrm{HBP} / \mathrm{PEG}$ 晶体的偏光显微镜照片与 PEG 晶体相似, 但黑十字 消光图像非常模糊, 多面体内部由大量径向发射生 长的针状晶体紧密堆积而成, 说明 $\mathrm{HBP} / \mathrm{PEG}$ 晶体的 主要生长方式与 $\mathrm{HBP}$ 相同, 均为针状晶体的一维生 长, HBP 起到了异相成核剂的作用. 这与用 Ozawa 方程处理得到的结果相符.

\section{4 .3 红外测试}

图 13 为 PEG、HBP/PEG、HBP 的红外光谱图. 可以看出, 加入超支化聚酯后, PEG 的羟基吸收峰 由 3431.6 移至 $3423.6 \mathrm{~cm}^{-1}$, 红移了 $8 \mathrm{~cm}^{-1}$. 这表明超 支化聚酯中的羰基与 PEG 的端羟基形成了氢键; 同
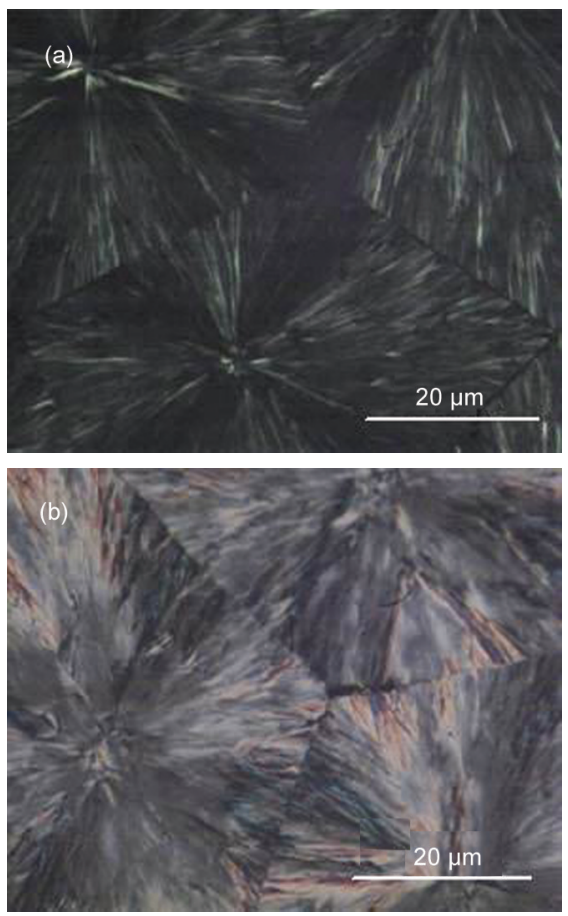

图 $1225^{\circ} \mathrm{C}$ 时 PEG (a)及 HBP/PEG (b) 的偏光显微镜照片 Fig.12 Polarizing microscope photos of PEG (a) and HBP/ PEG (b) at $25^{\circ} \mathrm{C}$ 


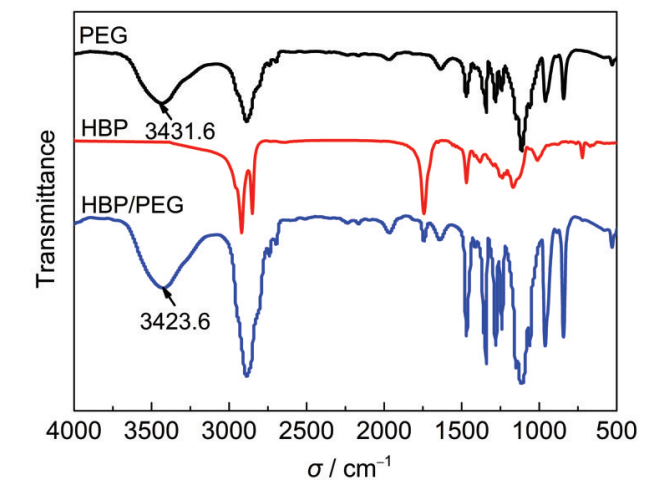

图 13 PEG、HBP、HBP/PEG 的红外光谱图 Fig.13 IR spectra of PEG, HBP, and HBP/PEG

时, 超支化聚酯较大的分子体积以及高度支化的结 构所导致的位阻效应也影响了 PEG大分子链向晶 核表面的排列堆砌过程, ${ }^{25}$ 使得 PEG 的结晶总速率 降低.

\section{4 结 论}

(1) PEG 和 HBP/PEG 的非等温结晶过程可用 Ozawa 和莫志深动力学方程描述, 与 Jeziorny 动力 学方程不符;

(2) 用 Ozawa 方程处理的非等温结晶动力学得 到的 Avrami 指数 $n$ 均为非整数, 且不同温度下的 $n$ 值和冷却结晶函数 $\lg k(T)$ 值相差较大, 说明 $\mathrm{PEG}$ 和 $\mathrm{HBP} / \mathrm{PEG}$ 的结晶机理复杂, 不同温度下存在着不同 的结晶机制, 超支化聚酯的加入改变了 PEG 晶体的 生长方式. 在相同的相对结晶度下, 用莫志深法处 理得到的 $\mathrm{HBP} / \mathrm{PEG}$ 体系的 $F(T)$ 值小于纯 $\mathrm{PEG}$ 的 $F(T)$ 值, 说明超支化聚酯的加入延缓了 PEG 的结晶;

(3) 超支化聚酯中的羰基与 PEG 的端羟基形成 的氢键以及超支化聚酯自身较大的分子体积和高 度支化的结构所导致的位阻效应是超支化聚酯延 缓 PEG 结晶的主要原因.

\section{References}

(1) Wang, W.; Shang, B. K.; Hu, S. Q.; Li, J. Chem. Propel Polymer. Mater 2004, 2 (5), 39. [王 伟, 尚丙坤, 胡少强, 李 洁. 化学推进剂与高分子材料, 2004, 2 (5), 39.]

(2) Chen, F. T.; Duo, Y. Q.; Luo, S. G.; Luo, Y. J.; Tan, H. M. Propel. Explos. Pyrotech. 2003, 28 (1), 7.

(3) Zhang, X. P.; Zhao, X. B.; Du, L.; Zheng, J. Acta Polym. Sin. 2004, No. 3, 388. [张小平, 赵孝须, 杜 否, 郑剑. 高分子 学报, 2004, No. 3, 388.]
(4) Byoung, S. M. Propel. Explos. Pyrotech. 2008, 33 (2), 131.

(5) Zhao, X. B.; Zhang, X. P.; Zheng, J.; Du, L. J. Propul. Technol. 2002, 23 (3), 252. [赵孝涁, 张小平, 郑 剑, 杜 否. 推进技 术, 2002, 23 (3), 252.]

(6) Jian, X. X.; Xiao, L. Q.; Zhou, W. L.; Xu, F. M. J. Solid Rocket Technol. 2008, 31 (4), 377 . [菅晓霞, 肖乐勤, 周伟良, 徐复 铭. 固体火箭技术, 2008, 31 (4), 377.]

(7) Liu, J. R.; Song, X. J.; Yang, Y. J. Solid Rocket Technol. 2010, 33 (1), 72. [刘晶如, 宋雪晶, 杨 寅. 固体火箭技术, 2010 , $33(1), 72$.

(8) Gao, C.; Yan, D. Y. Prog. Polym. Sci. 2004, 29 (3), 183.

(9) Vuković, J.; Steinmeier, D.; Lechner, M. D.; Jovanović, S.; Bozić, B. Polym. Degrad. Stab. 2006, 91 (8), 1903.

(10) Žagar, E.; Huskić, M.; Žigon, M. Macromol. Chem. Phys. 2007, 208 (13), 1379

(11) Dodds, J. M.; Luca, E. D.; Hutchings, L. R.; Clarke, N. J. Polym. Sci. Part B-Polym. Phys. 2007, 45 (19), 2762.

(12) Jena, K. K.; Raju, K. V.; Prathab, B.; Aminabhavi, T. M. J. Phys. Chem. B 2007, 111 (30), 8801.

(13) Fu, Q.; Liu, J. H.; Shi, W. F. Prog. Org. Coat. 2008, 63 (1), 100

(14) Ikladious, N. E.; Mansour, S. H.; Rozik, N. N.; Dirnberger, K.; Eisenbach, C. D. J. Polym. Sci. Part A-Polym. Chem. 2008, 46 (16), 5568 .

(15) Zhang, X. L. Polym. Int. 2011, 60 (2), 153.

(16) Žagar, E.; Žigon, M. Prog. Polym. Sci. 2011, 36 (1), 53.

(17) Tan, H. M.; Luo, Y. J. Hyperbranched Polymers; Chemical Industry Press: Beijing, 2005; pp 1-16. [谭惠民, 罗运军. 超支化聚合物. 北京: 化学工业出版社, 2005: 1-16.]

(18) Liu, J. R.; Chao, L. L.; Yu, Q.; Zhu, M. B.; Gu, C. M. Spectrosc Spectral. Anal. 2011, 31 (4), 983. [刘晶如, 巢玲玲, 俞 强, 朱梦冰, 顾春苗. 光谱学与光谱分析, 2011, 31 (4), 983.]

(19) Ozawa, T. Polymer 1971, 12 (3), 150.

(20) Jeziorny, A. Polymer 1978, 19 (10), 1142.

(21) Liu, J. P.; Mo, Z. S.; Qi, Y. C.; Zhang, H. F.; Chen, D. L. Acta Polym. Sin. 1993, No. 1, 1. [刘结平, 莫志深, 綦玉臣, 张宏 放, 陈东霖. 高分子学报, 1993, No. 1, 1.]

(22) Ren, M. Q.; Mo, Z. S.; Chen, Q. Y.; Zhang, H. F.; Song, J. B.; Zhang, H. L.; Sun, X. H. Acta Polym. Sin. 2005, No. 3, 374. [任敏巧, 莫志深, 陈庆勇, 张宏放, 宋剑斌, 张会良, 孙小红. 高分子学报, 2005, No. 3, 374.]

(23) Mo, Z. S. Acta Polym. Sin. 2008, No. 7, 656. [ [ 莫志深. 高分子 学报, 2008, No. 7, 656.]

(24) Bian, J.; Ye, S. R.; Feng, L. X. Chem. J. Chin. Univ. 2000, 21 (9), 1481. [边 界, 叶胜荣, 封麟先. 高等学校化学学报, 2000, 21 (9), 1481.]

(25) Liu, J. R.; Yu, Q.; Chen, K. J.; Xie, L. J. Chem. Eng. Chin. Univ. 2011, 25 (3), 447. [刘晶如, 俞 强, 陈科军, 谢 否. 高校化 学工程学报, 2011, 25 (3), 447.] 